\title{
Método de evaluación de la calidad del servicio de una unidad de atención al usuario en una empresa de servicio de agua en Colombia
}

\author{
Tomás J. Fontalvo ${ }^{\star}$, Enrique J. De la hoz-Dominguez ${ }^{2}$ y Efraín De la $\mathrm{Hoz}^{3}$ \\ (1) Universidad Libre, Programa de Ingeniería Industrial, Barranquilla, Colombia. \\ (correo-e: tomasjosefontalvo@gmail.com) \\ (2) Universidad Tecnológica de Bolívar, Facultad de Ingenierías, Programa Ingeniería Industrial, Campus de Ternera, \\ Cartagena, Colombia. (correo-e: edelahoz@utb.edu.co) \\ (3) Universidad de Cartagena, Facultad de Ciencias Económicas, Programa Administración Industrial, Campus Piedra \\ de Bolívar, Cartagena, Colombia. (correo-e: edelahozg@unicartagena.edu.co)
}

* Autor a quien debe ser dirigida la correspondencia.

Recibido Oct. 15, 2019; Aceptado Dic. 18, 2019; Versión final Ene. 22, 2020, Publicado Ago. 2020

\section{Resumen}

En esta investigación se propuso un método de evaluación de la calidad del servicio de una unidad de atención al usuario en una empresa de servicio de agua en Colombia. Apoyados en la fundamentación teórica asociada a la calidad del servicio y el concepto de Seis Sigma, se identificaron las dimensiones de calidad del servicio para consolidar la valoración de estos y analizar los resultados en términos de defectos por partes por millón (DPMO), nivel sigma y rendimiento. Se encontró que el desempeño de las dimensiones de calidad del servicio fue satisfactorio debido a los resultados obtenidos por todas las métricas calculadas. Los resultados demostraron que el centro de atención al usuario tuvo un desempeño excelente durante esta investigación. En conclusión, en esta investigación se aporta al sector de servicio un método de evaluación de la calidad del servicio en una empresa de servicios públicos por medio de Seis Sigma.

Palabras clave: calidad del servicio; seis sigma; empresa de servicio; servicio de aguas

\section{A method for evaluating the quality service of a user unit in a water service company in Colombia}

\begin{abstract}
This research study proposes a method to evaluate the quality of service of a user service unit in a water service company in Colombia. Based on the theoretical foundation associated with the quality of service and the Six Sigma concept, service quality dimensions were identified to consolidate their valuation and to analyze the results in terms of defects per parts per million (DPMO), sigma level, and performance. It was found that the performance of the service quality dimensions was satisfactory due to the results obtained in all calculated metrics. The results showed that the user's attention center had an excellent performance during this research study. In conclusion, the present study provides a service quality evaluation method to the service sector in a public service company by using Six Sigma.
\end{abstract}




\section{INTRODUCCIÓN}

Actualmente, las entidades de atención al usuario en organizaciones públicas son un espacio para captar, recibir, consolidar información y dar respuesta a solicitudes que realizan los usuarios, así como establecer acciones y actitudes efectivas que permitan cumplir las expectativas y establecer una relación eficiente con la sociedad, por lo cual, mantener un control de la calidad del servicio posibilita a estas entidades hacer un seguimiento detallado de las acciones así como detectar problemas y corregir rápidamente el desempeño. Es por lo anterior, que proponer un método de evaluación de la calidad del servicio en las unidades de atención al usuario resulta una necesidad, debido a que por medio de indicadores y niveles de desempeño que se consolidan mediante la aplicación de metodologías es posible llevar el servicio desde una perspectiva empírica a un enfoque medible y cuantificable. En el desarrollo de esta investigación se propone un método de evaluación de la calidad del servicio en una unidad de atención al usuario en una empresa de servicio de agua en Colombia para dos meses comprendidos en el 2018.

A lo largo de la historia, se ha desarrollado el tema de la calidad del servicio y de la importancia de su medición para que los resultados sean los deseados, es por esto que, definir indicadores que permitan llevar control de la calidad del servicio resulta de gran impacto en las empresas de servicio, debido a que, el no cumplimiento de ciertos niveles en estos indicadores puede llevar a las empresas a pagar multas o indemnizaciones a los usuarios afectados (Rojas-Ríos et al, 2016). Adicionalmente es importante señalar que, desafortunadamente muchos administradores de empresas de servicio dejan de lado las expectativas de los clientes respecto al servicio prestado, por concentrarse, entre otros aspectos, en la calidad del producto, la financiación del negocio o en actividades básicas de la operación de la empresa, inadvirtiendo que el cliente busca algo más que un buen producto (Causado-Rodriguez et al, 2019).

Desde el punto de vista de una empresa, la calidad del servicio puede verse simplemente como qué tan bien se ha llevado a cabo su desempeño operativo para servir a sus clientes (Phuong et al, 2019). No obstante, se ha demostrado el impacto que tiene en el funcionamiento de una unidad de servicio cuando existen estrategias que busquen su continuo mejoramiento. Sin embargo, relacionar dimensiones cualitativas a métricas requiere de un análisis profundo en su comportamiento, debido a que la calidad del servicio es uno de los principales determinantes de la satisfacción del cliente, es una construcción multidimensional, cuyas dimensiones difieren de un sector a otro (Nunkoo et al, 2019). Por lo anterior en esta investigación se evalúa el proceso de atención al usuario, enfocándose en valorar las dimensiones de calidad inherentes al servicio que brinda la organización y que son significativas para satisfacer a los usuarios de la empresa de servicios públicos de agua.

De acuerdo con lo planteado anteriormente, surgen los siguientes interrogantes sobre el desarrollo de la presente investigación: ¿Cómo evaluar las dimensiones de calidad del servicio en una unidad de atención al usuario?, ¿Cuál es la estructura, dimensiones de calidad, métricas cuantitativas a utilizar y criterios de desempeño del proceso de atención al usuario?, ¿Cómo valorar las dimensiones de calidad del proceso de atención del usuario por medio de las métricas de Seis Sigma? ¿Cuál fue el desempeño de las dimensiones de calidad del proceso de atención al usuario en los dos periodos? Con respecto a las preguntas previas, se estableció como objetivo principal a cumplir en la presente investigación, el proponer un método de evaluación de la calidad del servicio por medio de Seis Sigma para una unidad de atención al usuario en una empresa de servicio de agua en Colombia.

De manera más específica los objetivos, consecuentes con las preguntas problemas que se plantearon fueron los siguientes: i) Establecer una estructura, dimensiones de calidad, métricas cuantitativas a utilizar y criterios de desempeño del proceso de atención al usuario, ii) Valorar las dimensiones de calidad del proceso de atención del usuario por medio de las métricas de Seis Sigma, iii) Analizar el desempeño de las dimensiones de calidad del proceso de atención al usuario en los dos periodos v) Comparar el desempeño de las dimensiones de calidad valoradas por las métricas de Seis Sigma del proceso de atención al usuario en la empresa de servicio.

\section{OTROS ANTECEDENTES}

Se presentan dos aspectos de especial importancia para el desarrollo del tema: Calidad en el servicio público y Seis Sigma aplicado al servicio

\section{Calidad en el servicio público}

Hoy en día el sector de empresas privadas ha centrado sus esfuerzos en optimizar la calidad del servicio que presta, ofreciendo a la sociedad respuestas rápidas y una gestión del servicio eficiente, por lo tanto, el sector público se ve enfrentado a cumplir el prerrequisito de priorizar sus esfuerzos en mejorar continuamente la 
calidad del servicio; el autor Hrnjic (2016) menciona que las instituciones públicas y privadas que actualmente brindan servicios enfrentan enormes presiones económicas y escasez de capital, lo que ha llevado al dilema de cómo mantener satisfechos a sus clientes sin la necesidad de recurrir a reformas costosas; una manera de mantener el control de la calidad del servicio es por medio de métodos cuantitativos de evaluación

Es por lo anterior que se han llevado a cabo distintos estudios que buscan estrategias para mejorar los servicios que se prestan en las organizaciones públicas. En el estudio llevado a cabo por Rita et al (2019) se desarrollaron nuevos conocimientos para comprender mejor las dimensiones más importantes de la calidad del servicio que tienen un impacto en la satisfacción del cliente, la confianza del cliente y el comportamiento del cliente. En un estudio realizado por los autores Amrapala y Choocharukul (2019) donde evaluaron la calidad del servicio de una entidad de servicio público de transporte por medio de una perspectiva cualitativa, reveló dimensiones de la calidad del servicio inherentes a dicho servicio, que explican la percepción del usuario y las prioridades con respecto al servicio, entre las cuales se encuentran factores de actitud, que incluyen confiabilidad, ambiente, comodidad y conveniencia. Similarmente, Yildiz y Kara (2015) señalan que, aunque las instituciones públicas no enfrentan las condiciones del mercado como empresas comerciales, para aquellas instituciones que no brindan servicios de calidad, sus esfuerzos efectivos para avanzar con los objetivos de la organización no tendrán buenos resultados.

Las metodologías empleadas para medir la calidad del servicio público han sido variadas, en un estudio desarrollado por (Aceneth, 2018) se empleó el modelo de SERVPERF, el cual se adaptó a los servicios con el propósito de medir las percepciones de calidad asociadas con el servicio público. De igual forma, Causado et al (2019) han mostrado la pertinencia del uso de modelos de la calidad en el servicio, para evaluar dimensiones de calidad asociadas con la satisfacción de los usuarios lo que al final se refleja en la mejora de los procesos de prestación del servicio.

\section{Seis Sigma aplicado al servicio}

La metodología de seis sigma se ha aplicado exitosamente en distintos sectores, incluyendo el de manufactura y el de servicio, debido a los beneficios que presenta en la evaluación estadística de la calidad representando el factor cuantitativo de la presente investigación, esta herramienta proporciona enfoques basados en evidencia que apoyan el mejoramiento de la calidad en la práctica (Davies et al, 2019). Así mismo en una investigación desarrollada por (López y Michelena, 2014) mencionan que esta metodología tiene como intencionalidad trabajar de forma puntual sobre los procesos, para de esta manera reducir los defectos y generar mejoras asociadas con el bienestar económico mediante la eliminación sistemática de defectos.

En distintas investigaciones previas se ha demostrado las ventajas que ha traído la implementación de la metodología seis sigma en el sector de servicios, como por ejemplo en la llevada a cabo en (Alkoot, 2019) quienes encontraron que la implementación de esta técnica cuantitativa lleva a diagnosticar si existen problemas y establece el camino para encontrar las causas que debemos tratar para obtener mejoras. Así mismo, esta técnica proporciona un marco fundamental para mejorar la eficiencia, reduciendo la variabilidad (Bonome, 2018) lo cual permite una estandarización del nivel de servicio que se le proporciona al cliente

En la misma línea de ideas, en el estudio desarrollado por (Miniati et al., 2015) donde aplicaron esta herramienta en un centro de servicios públicos, se pudo proporcionar información importante para la gestión de decisiones, como la evaluación de la carga de trabajo y la planificación específica de la estación de trabajo. Similarmente en la investigación llevada a cabo por (Hynes et al, 2019) en el contexto de servicio público médico, y por medio de la aplicación de los principios Lean Seis Sigma en la disminución de los tiempos de espera se encontró una mejora significativa de 3.74 días a 1.88 días. Lo cual según Tagge et al (2017) permite evidenciar que un rediseño coordinado de mejora de procesos puede mejorar significativamente sin la necesidad de preseleccionar servicios específicos o incorporar nuevo personal o tecnología.

\section{METODOLOGÍA}

Como fuente primaria de información se partió de los datos suministrados por el proceso de atención al usuario de la empresa de servicio de agua, sobre la cual se calcularon las métricas Seis Sigmas $(Z)$ y $(Y)$, las cuales permitieron valorar las dimensiones del servicio. La información utilizada para la aplicación del modelo se tomó por medio de revisión documental, directamente de la base de datos de los reportes realizados por la unidad de atención del cliente en los periodos correspondientes, donde se plasmaron todas las solicitudes recibidas, así como el cumplimiento de las distintas fases del servicio prestado. La validez y confiabilidad de la información suministrada se fundamenta en que se realizó con la información total de las dimensiones evaluadas en los 11 meses, por parte de los encargados de la gestión de control de calidad de la empresa de servicio de agua, los cuales presentaron una posición imparcial con respecto a los resultados de los datos encontrados, así como su objetivo principal de establecer una evaluación objetiva. Este estudio investigativo 
se llevó a cabo mediante una perspectiva de carácter evaluativa, por lo que, se realizó un análisis empírico del servicio prestado por la unidad de atención al usuario en dos meses consecutivos del año 2018, marzo y abril, a partir de un análisis general de las acciones efectuadas en los dos periodos se elaboró un análisis racional que permitió identificar los criterios de valoración de la calidad del servicio de la unidad de atención al usuario.

La fundamentación en la cual se basó el estudio para determinar las dimensiones de calidad fue principalmente en la naturaleza del servicio de la unidad de atención al cliente, para la cual era fundamental establecer dimensiones de calidad que representaran la esencia de su gestión tal como la organización de la respuesta de la solicitud y la recepción efectiva de la solicitud del usuario. Similarmente, cuando se estructuró la información proveniente directamente de la unidad de atención al cliente, se pudo observar que para establecer si el servicio fue admisible o no, fue necesario evaluar cualitativamente el proceso basados en unas ciertas etapas inherentes al trabajo, las cuales se tomaron en cuenta para su evaluación cuantitativa por medio de Seis Sigma y el presente método. Adicionalmente, se estudiaron dimensiones aplicadas en contextos similares, entre ellos Raviadaran et al. (2019) quienes llegaron a definir dimensiones tales como la fiabilidad, la capacidad de respuesta, la comunicación, el acceso y la seguridad para conducir la satisfacción del cliente; las cuales son coherentes con las tenidas en cuenta en la presente investigación. Cabe resaltar que las dimensiones del servicio propuestas son criterios subjetivos que se relacionan directamente a el contexto donde se aplican por lo que su fundamentación depende mayormente de la naturaleza del servicio que se estudia.

A partir de esta categorización de las dimensiones de calidad se procedió a contextualizar las métricas referentes a la metodología de seis sigma con los criterios de evaluación con el objetivo de llevar las distintas dimensiones de calidad cualitativas del servicio a indicadores medibles. Seguidamente, se efectuó la valoración de las métricas de seis sigma para determinar los resultados de la evaluación de las dimensiones de calidad, obteniendo también el rendimiento para cada uno de los periodos correspondientes. Después haber calculado las métricas, como son niveles de rendimiento y nivel sigma del servicio fue posible realizar un análisis comparativo de los dos meses en cuestión con el objetivo de evaluar la evolución de la calidad del servicio y verificar el desempeño que la empresa de servicio de agua efectuó en cada mes. En la Figura 1 se presenta el proceso de atención al usuario de la empresa de servicios de agua, así como las dimensiones de calidad, su descripción y las métricas que se utilizaron para evaluar. Para esta investigación se consideró un buen desempeño de las dimensiones calidad del servicio cuando el rendimiento $(Y)$ estuvo sobre $95 \%$. Y el nivel sigma (Z) superó 3.

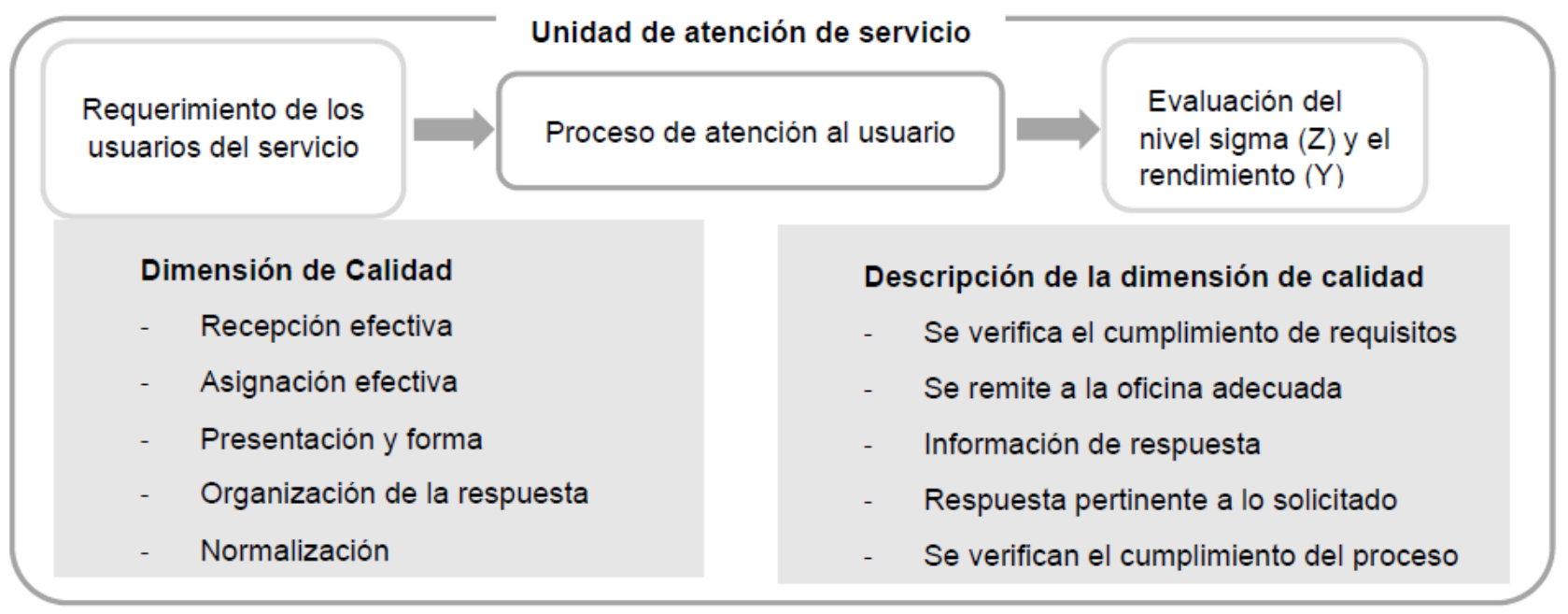

Fig.1: proceso de prestación del servicio en dimensiones de calidad y su descripción

Debido a la naturaleza de la investigación, se emplearon análisis descriptivos, explicativos y evaluativos de la calidad, así mismo, por medio de la aplicación de la metodología de seis sigma fue posible llevar la evaluación de la calidad del servicio desde un enfoque conceptual y cualitativo hasta un enfoque objetivo y cuantitativo, permitiendo a la empresa de servicio de agua tener control de la gestión del servicio que ofrece, utilizando información objetiva, soportada por los procesos históricos de atención a los usuarios. Las métricas calculadas fueron el nivel sigma Z, los defectos de partes por millón (DPMO) y el rendimiento (Y). En la Figura 2 se plasmó el método de evaluación de la unidad de atención al usuario y sus dimensiones de calidad. 


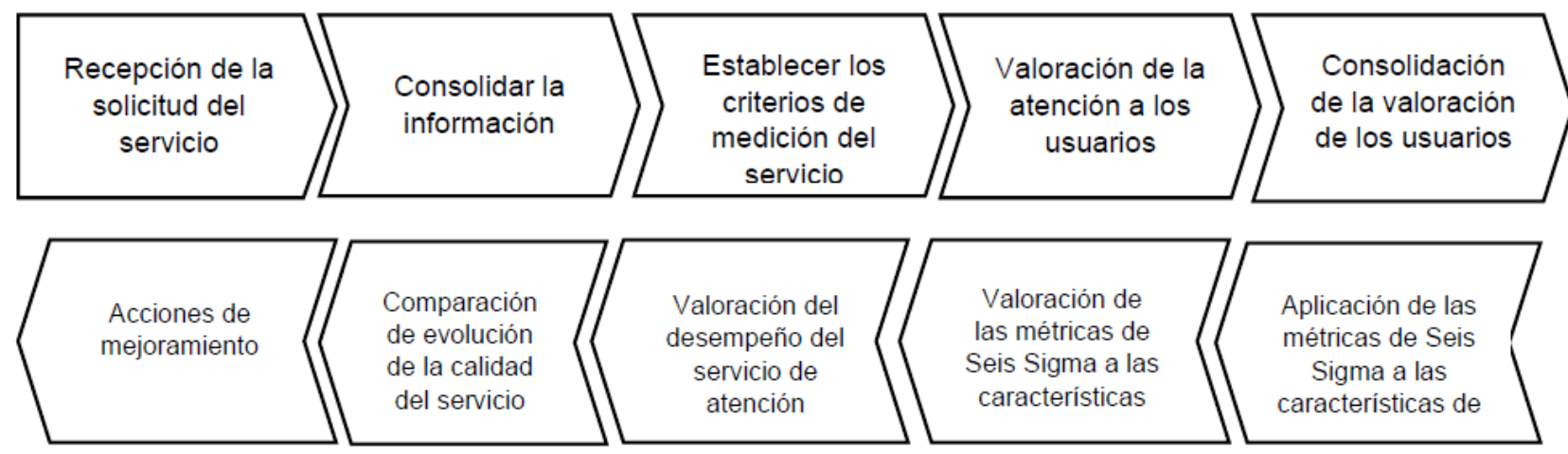

Fig.2: Método de Evaluación de la calidad del servicio por medio de Seis Sigma a unidad de atención al usuario

El método que se propone en la presente investigación se diferencia de otras estrategias debido a que se apoya en la fundamentación teórica asociada a la calidad del servicio, y la técnica estadística de Seis Sigma, la cual le permite tener el control de la variabilidad del proceso de manera que sea posible disminuir posibles fallas en este. Así mismo, por medio de un enfoque empírico y racional fue posible tener una perspectiva objetiva durante el desarrollo del método. Otro factor diferenciador es que el presente método parte de los datos reales del proceso de servicio, lo cual incluye el papel del empleado cuando se presenta una solicitud, la gestión de la unidad de atención al cliente, y el tipo de respuesta que se da a la solicitud, por lo que es posible evaluar la calidad de este basados en la propia gestión de la organización y no basados en la percepción subjetiva del cliente.

\section{RESULTADOS Y DISCUSIÓN}

Considerando las intencionalidades de este estudio a continuación se explican en detalle la forma de valorar y analizar las dimensiones de calidad del proceso de atención del usuario por medio de las métricas de Seis Sigma y la forma como se calcula y analiza el desempeño de las dimensiones de calidad del proceso de atención al usuario en los dos periodos. En la Tabla 1 se presenta la información proporcionada por la unidad de atención al usuario describiendo las dimensiones de calidad evaluadas en la unidad de atención.

Tabla 1: Información del servicio categorizada en los criterios de evaluación de la calidad

\begin{tabular}{|c|c|c|c|c|c|c|c|c|c|c|}
\hline \multirow{2}{*}{$\begin{array}{l}\text { Desempeño } \\
\text { del servicio }\end{array}$} & \multicolumn{10}{|c|}{ Dimensiones de calidad evaluadas } \\
\hline & \multicolumn{2}{|c|}{ Recepción efectiva } & \multicolumn{2}{|c|}{$\begin{array}{l}\text { Asignación } \\
\text { efectiva }\end{array}$} & \multicolumn{2}{|c|}{$\begin{array}{l}\text { Presentación y } \\
\text { forma }\end{array}$} & \multicolumn{2}{|c|}{$\begin{array}{l}\text { Organización de } \\
\text { la respuesta }\end{array}$} & \multicolumn{2}{|c|}{$\begin{array}{c}\text { Normalización del } \\
\text { servicio }\end{array}$} \\
\hline Periodo & Marzo & Abril & Marzo & Abril & Marzo & Abril & Marzo & Abril & Marzo & Abril \\
\hline $\begin{array}{l}\text { Servicio } \\
\text { admisible }\end{array}$ & 2322 & 4338 & 314 & 614 & 484 & 1166 & 974 & 2274 & 790 & 1796 \\
\hline $\begin{array}{c}\text { Servicio } \\
\text { inadmisible }\end{array}$ & 168 & 244 & 20 & 208 & 20 & 34 & 34 & 126 & 34 & 208 \\
\hline
\end{tabular}

En la Tabla 1, se puede observar el número de servicio prestados por la unidad de atención al usuario categorizada en servicios admisibles e inadmisibles, el cual se clasificó según los criterios establecidos en los procesos de normalización de calidad de la empresa de servicio de agua. A partir de esta información fue posible calcular las métricas de calidad relacionadas a Seis Sigma para estudiar la evolución del servicio y comparar rendimientos entre los dos meses en cuestión.

\section{Métricas de Seis Sigma para evaluar la calidad del servicio}

A partir de la información presentada en la Tabla1 fue posible aplicar el enfoque de mejoramiento de procesos de Seis Sigma; el cual se desarrolló mediante un estudio detallado de los conceptos de Seis Sigma como las oportunidades de error, la cantidad de servicios prestados y el número de servicios inadmisibles, para así obtener el rendimiento global del proceso de atención al usuario; al realizar la contextualización de los parámetros de esta metodología con los criterios de evaluación, fue posible valorar las métricas de Seis Sigma adaptando las dimensiones de calidad del proceso de atención a los usuarios al esquema de medición Seis Sigma, obteniendo la siguiente relación de variables: U: Cantidad de servicios; O: Oportunidad de error; n: número de servicios inadmisibles; Y: Rendimiento del proceso de atención al usuario; DPMO: Defectos de partes por millón 
Las ecuaciones que se emplearon para calcular las métricas de defectos en partes por millón de oportunidades (DPMO), el rendimiento (Y) y el nivel Sigma (Z), se presentan a continuación

$$
\begin{aligned}
& P M O=\frac{n}{t} \times 1.000 .000=\frac{n}{U x O} \times 1.000 .000 \\
& Y=\left(1-\frac{n}{U \times O}\right) \\
& Z=\sqrt{ }(29.37-2.221 \times \ln (D P M O)+(0.8406)
\end{aligned}
$$

Las métricas de las dimensiones de calidad del servicio de la unidad de atención al usuario de la empresa de servicio de agua para los meses de marzo y abril se muestran en la Tabla 2 y 3 respectivamente, desarrollando para cada dimensión del servicio los cálculos respectivos asociados al enfoque Seis Sigma y obteniendo el rendimiento promedio del sistema en general.

En la Tabla 2, se presentan los resultados de las métricas de Seis Sigma para cada una de las dimensiones de calidad evaluadas en el mes de Marzo, se halló que el criterio con mayor nivel sigma fue el de 'Estructura Comunicado' y el que tuvo menor valoración fue el de 'Presentación y forma', sin embargo, todas los criterios estuvieron por encima del valor crítico de $Z=3$ por lo tanto, en términos generales la evaluación del servicio demostró que todos los criterios de evaluación llevan un proceso satisfactorio. Similarmente, en la Tabla 3 se encuentran los resultados de las métricas de las dimensiones de calidad del servicio para la unidad de atención al usuario en el mes de abril. Se encontró que el criterio que obtuvo mayor nivel sigma fue el de 'Recepción efectiva' y aquel que estuvo por debajo del resto fue el de 'Asignación efectiva'.

Tabla 2. Cálculo de las métricas de Seis Sigma para las dimensiones de calidad en el mes de marzo

\begin{tabular}{|c|c|c|c|c|c|}
\hline \multirow{2}{*}{$\begin{array}{c}\text { Métricas de Seis } \\
\text { Sigma }\end{array}$} & \multicolumn{5}{|c|}{ Dimensiones de calidad evaluadas } \\
\hline & $\begin{array}{l}\text { Recepción } \\
\text { efectiva }\end{array}$ & $\begin{array}{c}\text { Asignación } \\
\text { efectiva }\end{array}$ & $\begin{array}{l}\text { Presentación y } \\
\text { forma }\end{array}$ & $\begin{array}{c}\text { Organización } \\
\text { de la } \\
\text { respuesta }\end{array}$ & $\begin{array}{c}\text { Normalización } \\
\text { del servicio }\end{array}$ \\
\hline $\begin{array}{l}\text { Variables de Seis } \\
\text { Sigma }\end{array}$ & $\begin{array}{l}\text { U: } 4582 \\
\text { O: } 6 \\
n: 244\end{array}$ & $\begin{array}{l}\text { U: } 822 \\
\text { O: } 5 \\
n: 208\end{array}$ & $\begin{array}{l}\text { U: } 1200 \\
\text { O: } 3 \\
n: 34\end{array}$ & $\begin{array}{l}\text { U: } 2400 \\
\text { O: } 4 \\
n: 126\end{array}$ & $\begin{array}{l}\text { U: } 2004 \\
\text { O: } 4 \\
n: 208\end{array}$ \\
\hline DPMO & 8875,30 & 50608,27 & 9444,44 & 13125 & 25948,10 \\
\hline Nivel Sigma Z & 4,63 & 4,44 & 4,62 & 4,58 & 4,517 \\
\hline Rendimiento $\mathrm{Y}$ & 0,99 & 0,94 & 0,99 & 0,98 & 0,97 \\
\hline $\begin{array}{l}\text { Rendimiento } \\
\text { promedio }\end{array}$ & 0,97 & & & & \\
\hline
\end{tabular}

\begin{tabular}{|l|l|l|l|l|l|}
\hline \multirow{2}{*}{\begin{tabular}{l} 
Sétricas de Seis \\
\cline { 2 - 6 }
\end{tabular}} & $\begin{array}{l}\text { Dimensiones de calidad evaluadas } \\
\text { efectiva }\end{array}$ & $\begin{array}{l}\text { Asignación } \\
\text { efectiva }\end{array}$ & $\begin{array}{l}\text { Presentación y } \\
\text { forma }\end{array}$ & $\begin{array}{l}\text { Organización } \\
\text { de } \\
\text { respuesta }\end{array}$ & $\begin{array}{l}\text { Normalización } \\
\text { del servicio }\end{array}$ \\
\hline $\begin{array}{l}\text { Variables de Seis } \\
\text { Sigma }\end{array}$ & $\begin{array}{l}\text { U: } 2490 \\
\text { O: } 6 \\
\mathrm{n}: 168\end{array}$ & $\begin{array}{l}\text { U: } 334 \\
\text { O: } 5 \\
\mathrm{n}: 20\end{array}$ & $\begin{array}{l}\text { U: } 504 \\
\text { O: } 3 \\
\mathrm{n}: 20\end{array}$ & $\begin{array}{l}\text { U: } 108 \\
\text { O: } 4 \\
\mathrm{n}: 34\end{array}$ & $\begin{array}{l}\text { U: } 824 \\
\text { O: } 4 \\
\mathrm{n}: 34\end{array}$ \\
\hline DPMO & 11244,97 & 11976,04 & 13227,51 & 8432,53 & 10315,53 \\
\hline Nivel Sigma Z & 4,60 & 4,59 & 4,58 & 4,63 & 4,61 \\
\hline Rendimiento Y & 0,98 & 0,98 & 0,98 & 0,99 \\
\hline $\begin{array}{l}\text { Rendimiento } \\
\text { promedio }\end{array}$ & 0,98 & & & \\
\hline
\end{tabular}

Tabla 3. Cálculo de las métricas de Seis Sigma para las dimensiones de calidad en el mes de abril

Como hallazgo fundamental se puede aseverar por la evidencia que el centro de atención al usuario presta un servicio muy bueno, considerando que las métricas de seis sigmas valoradas a las dimensiones de calidad, como son rendimiento $Y$ y nivel sigma $Z$ para los dos meses objeto de estudio alcanzaron valores para los dos meses de sigma $Z$ sobre 4, lo cual es muy bueno. $Y$ consecuentemente con esto el nivel de rendimiento promedio para los dos periodos fue de $98 \%$ para el primero periodo y $97 \%$ para el segundo periodo, lo cual 
es excelente, considerando que supera el 95\%. Como hallazgo de esta investigación también se evidenció que no mejoró el desempeño en todos los criterios del mes de marzo al mes de abril, es decir, para algunos criterios su desempeño fue de mejora como el de 'Presentación y forma' y para otras dimensiones se evidenció que estas no evolucionaron en los dos periodos.

En estudios similares donde se aplicaron las métricas de Seis Sigma para valorar la calidad del servicio se encontraron resultados coherentes con los obtenidos en esta investigación; un ejemplo es la investigación llevada a cabo por los autores (Purnama et al., 2018) se encontró que al momento de evaluar la calidad de un proceso se dio una disminución en los defectos por partes por millón lo que conllevó a una mejora del nivel Sigma Z de 3.61 a 3.86, lo que conllevó a centrar los esfuerzos en tratar de disminuir los defectos del proceso. Sin embargo, en el proceso de aplicación de la gestión Seis Sigma, también deben tenerse en cuenta los siguientes puntos: el valor de la satisfacción del cliente en diferentes períodos, cada mejora debe encontrar los factores más críticos que afectan la satisfacción del cliente, mejorando cada vez para un elemento (Zhuo, 2019)

En esta misma línea de ideas, en un estudio realizado por los autores O'Donnell, R. y Kessler (2018) afirman que las estrategias y técnicas de Seis Sigma parecen adecuadas para satisfacer la demanda emergente de una mejor calidad de atención, eficiencia, resultados, procesos o flujos de trabajo y salud laboral del proveedor.

Siguiendo con este razonamiento, en el estudio realizado por Arunkumar y Dillibabu (2016), utilizando una metodología basada en Seis Sigma, se llevó a cabo un análisis para eliminar los requisitos de los procesos sin valor e implementar la funcionalidad principal para satisfacer las expectativas del cliente. Cuando se revisa otra investigación (Shamsuzzaman et al, 2018) en otros contextos que aplicaron las métricas de seis sigmas obteniendo incremento del nivel sigma de 0.73 a 2.66, se puede aseverar que el desempeño en nuestro contexto fue muy superior. Considerando que el valor de las métricas de rendimiento $(Y)$ y nivel sigma $(Z)$ de las dimensiones de calidad alcanzado en nuestra investigación obtuvo un nivel sigma Z sobre 4. Es importante señalar que otros autores han utilizado métricas similares para evaluar el desempeño de un servicio en diferentes contextos (Fontalvo et al., 2010; Fontalvo y Cardona, 2000)

\section{CONCLUSIONES}

Como resultado de este trabajo de investigación se puede concluir lo siguiente:

1) A pesar de que los indicadores de rendimiento $(Y)$ y el nivel sigma $(Z)$ no mostraron un aumento de un periodo a otro, cuando se analizan los niveles alcanzados en la evaluación de las dimensiones de calidad se puede observar que estuvieron por encima del indicador deseado, por lo tanto, se puede aseverar que el centro de atención al usuario presenta un excelente servicio.

2) Como resultado de la integración de los conceptos de calidad del servicio, dimensiones de calidad, métricas de seis sigmas, es decir rendimiento y nivel sigma, se pudo evidenciar la utilidad y pertinencia de esta articulación para valorar un proceso de prestación del servicio en una unidad de atención al usuario.

3) El aporte significativo del estudio consiste en la presentación de un método replicable y reproducible en otros contextos empresariales de gestión de la calidad del servicio, generando un esquema comparable del nivel de desempeño organizacional con los estándares Seis Sigma.

4) Se aporta a la comunidad científica y a todas las empresas y organizaciones de servicios, estructuras, dimensiones de calidad, métricas de seis sigma ( $\mathrm{Y}$ y Z $\mathrm{Z}$, criterios de desempeño, que permiten valorar cualquier tipo de servicio, con lo cual tomar decisiones para la mejora y satisfacción de los usuarios.

\section{REFERENCIAS}

Aceneth, M., Análisis del impacto del proceso de tercerización en la percepción de calidad del servicio público domiciliario de energía eléctrica en el municipio de Quibdó, Revista CES Derecho, 9(1), 34-58 (2018)

Alkoot, F.M., Using 6 sigma to improve outcomes of higher education institutes. https://doi.org/10.18178/ijiet.2019.9.1.1171, International Journal of Information and Education Technology, 9(1), 46-50 (2019)

Amrapala, C. y Choocharukul, K., Perceived service quality and commuter segmentation of informal public transport service. https://doi.org/10.4186/ej.2019.23.6.1, Engineering Journal, 23(6) (2019)

ArunKumar, G. y Dillibabu, R., Design and Application of New Quality Improvement Model: Kano Lean Six Sigma for Software Maintenance. https://doi.org/10.1007/s13369-015-1933-1, Project Arab J Sci Eng, 41, 997-1014 (2016) 
Bonome, L., Godinho, M., Fredendall, L. y Gómez, F., Six sigma and lean six sigma in the food industry: A systematic literature review. https://doi.org/10.1016/j.tifs.2018.10.002, Trends in Food Science \& Technology, 82, 122-133 (2018)

Causado, E., Charris, A.N. y Guerrero, E.A., Mejora Continua del Servicio al Cliente Mediante ServQual y Red de Petri en un Restaurante de Santa Marta, Colombia. https://doi.org/0.4067/S0718-07642019000200073, información tecnológica, 30 (2), 73-84 (2019)

Causado-Rodriguez, E., Charris, A.N. y Guerrero, E.A., Mejora Continua del Servicio al Cliente Mediante ServQual y Red de Petri en un Restaurante de Santa Marta, Colombia. https://doi.org/10.4067/S0718-07642019000200073, Información tecnológica, 30(2), 73-84 (2019)

Davies, C., Lyons, C. y Whyte, R. Optimizing nursing time in a day care unit: Quality improvement using Lean Six Sigma methodology. https://doi.org/10.1093/intqhc/mzz087, International journal for quality in health care: journal of the International Society for Quality in Health Care, 31(1), 22-28 (2019)

Fontalvo, T. y Cardona, D., La cadena de suministro: un enfoque práctico para el diseño e implementación del modelo SCOR, Corporación para la gestión del conocimiento ASD 2000, (2000)

Fontalvo, T., De La Hoz, E. y Cardona, D., Diseño de un plan de mejoramiento para la cadena de suministro de la empresa Drolitoral SA, Aplicando el Modelo SCOR, 6(1), 33-53,(2010)

Hrnjic, A. The transformation of higher education: evaluation of CRM concept application and its impact on student satisfaction. https://doi.org/10.1007/s40821-015-0037-x, Eurasian Bus Rev, 6, 53-77 (2016)

Hynes, J.P, Murray, A.S y otros cinco autores, Use of Lean Six Sigma methodology shows reduction of inpatient waiting time for peripherally inserted central catheter placement, Clinical Radiology, 74 (9), 733.e5-733.e9 (2019)

López, Z. y Michelena, E., Mejora del proceso de prestación del servicio en una instalación de servicios gastronómicos, Ingeniería Industrial, 35(1), 34-44 (2014)

Miniati R., Frosini, F. y otros seis autores, Experience of Lean Six Sigma Quality Approach to Hospital Laboratory Services, 6th European Conference of the International Federation for Medical and Biological Engineering, 45, 609-612 (2015)

Nunkoo, R., Teeroovengaduma, V., Ringlee, C. y Sunnasseeg, V., Service quality and customer satisfaction: The moderating effects of hotel star rating. https://doi.org/10.1016/j.ijhm.2019.102414, International Journal of Hospitality Management, (2019)

O'Donnell, R. y Kessler, R., Quality Improvement, Performance Management, and Outcomes: Lean Six Sigma for Integrated Behavioral Health, Training to Deliver Integrated Care, Springer, Cham 79-101 (2018)

Phuong, T., Grant, D. y Menachof, D., Exploring logistics service quality in Hai Phong, Vietnam. https://doi.org/10.1016/j.ajsl.2019.12.001, The Asian Journal of Shipping and Logistics (2019)

Purnama, D.A., Shinta, R.C. y Helia, V.N. Quality improvements on creative industry by using Six Sigma: A study case. https://doi.org/10.1051/matecconf/201815401088, MATEC Web of Conferences, 154(01087), (2018)

Raviadaran, H., Dastane, O., Ma'arif, M.Y. y Mohd-Satar, N. S., Impact of Service Quality Dimensions on Internet Banking Adoption, Satisfaction and Patronage, International Journal of Management, Accounting and Economics, 6(10), 709-730 (2019)

Rita, P., Oliveira, T. y Farisa, A., The impact of e-service quality and customer satisfaction on customer behavior in online shopping. https://doi.org/10.1016/j.heliyon.2019.e02690, 5(10), (2019)

Rojas-Ríos, L.F., López-Lezama, J.M. y Muñoz-Galeano, N., Asignación Óptima de Presupuesto para Mejoramiento de la Calidad del Servicio en Sistemas de Distribución usando Algoritmo Genético No-Dominado II (NSGA-II) y un Algoritmo Memético. https://doi.org/10.4067/S0718-07642016000100013, Información tecnológica, 27(1), 115-126 (2016).

Shamsuzzaman, M., Alzeraif, M., Alsyouf, I. y Khoo, M.B.C., Using Lean Six Sigma to improve mobile order fulfilment process in a Telecom Service Sector. https://doi.org/10.1080/09537287.2018.1426132, Production Planning and Control, 29 (4), 301-314 (2018)

Tagge, E., Arul, S. y otros tres autores, Improving operating room efficiency in academic children's hospital using Lean Six Sigma methodology, https://doi.org/10.1016/j.jpedsurg.2017.03.035, Journal of Pediatric Surgery, 52(6), 1040-1044 (2017)

Yildiz, S.M. y Kara, A., Developing Alternative Measures for Service Quality in Higher Education: Empirical Evidence from the School of Physical Education and Sports Sciences, Proceedings of the 2009 Academy of Marketing Science (AMS) Annual Conference, 185-185 (2015)

Zhuo, Z., Research on using Six Sigma management to improve bank customer satisfaction, https://doi.org/10.1186/s40887-019-0028-6, Int J Qual Innov, 5(3), (2019) 